\title{
TASK-ORIENTED PHYSICAL EXERCISE USING POSTURAL RE- ALIGNMENT WITH BODY WEIGHT SUPPORT IN CHRONIC STROKE
}

\author{
R. SAGGINI ${ }^{1}$, A. DI STEFANO ${ }^{2}$, F. CAPOGROSSO ${ }^{2}$, S.M. CARMIGNANO ${ }^{3}$, S. D'ETTOLE ${ }^{3}$, \\ L. DI PANCRAZIO ${ }^{4}$, G. BARASSI ${ }^{5}$, R.G. BELLOMO ${ }^{4}$ \\ ${ }^{1}$ Department of Neuroscience and Imaging, "G. D'Annunzio" University, Chieti, Italy \\ ${ }^{2}$ School of Specialties in Physical Medicine and Rehabilitation, "G. d'Annunzio" University, Chieti, Italy \\ ${ }^{3}$ CUMS "G. D'Annunzio" University, Chieti, Italy \\ ${ }^{4}$ Department of Medicine and Science of Aging, "G. D'Annunzio" University, Chieti, Italy \\ ${ }^{3}$ Section of Physical Medicine and Rehabilitation, "G. D'Annunzio" University, Chieti, Italy
}

\begin{abstract}
The recovery of functional gait is the main target for subjects who had suffered a stroke. The methods designed to improve balance and gait appear to be essential for skills and autonomy and to reduce the costs of assistance. The aim of our study was to evaluate the improvement of stroke victims in the chronic phase through the rehabilitation of gait, balance and posture using postural re-alignment with specific body weight support. Our study includes 20 subjects with residual hemiparetic gait after stroke. Evaluation with international rating scales, gait analysis and stabilometric test was carried out at the beginning and after the $1^{\text {st }}$ and the $3^{\text {rd }}$ month of therapy; a follow-up control was made 3 months after the end of the rehabilitation program. All subjects underwent the rehabilitation protocol with Dynamic Antigravity Postural System 2 times a week for 3 months and were also treated with high efficiency focused acoustic waves (ViSS) to increase strength and muscular endurance $(300 \mathrm{~Hz})$ or to reduce spastic hypertonia $(200-120 \mathrm{~Hz})$; we also made neuromuscular stimulation with Manual Therapy to deactivate trigger points in muscles, tendons, and ligaments and to lengthen the chronically shortened muscles and balance muscle groups. The study shows a significant improvement in gait and balance with the persistence of results at the follow-up 3 months after the end of treatment. The subjects showed an increase in walking speed, greater stability and a consequent reduction of sedentary lifestyle with less risk of complications or recurrence. In conclusion this rehabilitation program is efficient for posture and walking quality.
\end{abstract}

